\title{
Mobile Emergency Response Application Using Geolocation for Command Centers
}

\author{
Jethro B. de Guzman, Ritz Carlo C. de Guzman, and Engr. Remedios G. Ado
}

\begin{abstract}
This paper introduces Mobile Emergency Response Application using Geolocation for Command Centers. It is a combination of a mobile and web application for responding to emergency requests for ambulance, fire truck and police by people in a certain area or city. The mobile application would detect user's current location through geolocation and sends to the web application deployed in a command center the name, age, mobile number and location of the user for easily dispatching of emergency units.
\end{abstract}

Index Terms-Command center, emergency response, geolocation.

\section{INTRODUCTION}

The actions and responses taken in the initial minutes of an emergency are critical. These life threatening events may happen any moment. Being always prepared and ready can save lives. A call for help to public emergency services that provides full and accurate information will help the dispatcher send the right responders and equipment.

Environmental emergencies are incidents or events that threaten public safety, health, and welfare and include hurricanes, floods, wildfires, industrial plant explosions, chemical spills, acts of terrorism, and others [1]. Emergency response is the organizing, coordinating, and directing of available resources in order to respond to the event and bring the emergency under control. The goal of this coordinated response is to protect public health by minimizing the impact of the event on the community and the environment.

One of the most popular and well known emergency systems in the world is America's 911. The system was designed to provide a universal, easy-to-remember number for people to reach police, fire or emergency medical assistance from any phone in any location, without having to look up specific phone numbers [2]. The technology, regulations and funding that make the system possible are largely based on the technology that existed at the time 911 was first implemented during the late 1960s -i.e., wired phones in residences and businesses.

The Philippines created its version of 911 called PATROL 117. Patrol 117 is the national and official emergency hotline number of the Philippines [3]. It aims to establish an easy recall number that can be accessed by anyone, anytime, anywhere in the Philippines in cases of emergencies, as well as to monitor the efficiency of its responders' network. It however, does not compete with other locally established emergency numbers or with local

Manuscript received December 5, 2013; revised March 12, 2014.

The authors are with the Polytechnic University of the Philippines, Philippines (e-mail: jethdeguzman@gmail.com). responders, but complements their local operations.

There are also a number of mobile applications available in smart phones that are beneficial in disaster response [4]. Among these are GPS technology, which can be used in the tracking of rescuers and resources, the translator, which can be used for communication, and the field examiner, which can be used to send information to headquarters for assessment of damages. Indeed, the use of a smart phone in a disaster management system is advantageous.

Command Centers handle certain communities. The release of a smart phone app increases the participation and preparation of the community in certain disasters. Ref. [5] Community-based disaster risk management is a process in which at-risk communities are actively engaged in the identification, analysis, treatment, monitoring and evaluation of disaster risks in order to reduce their vulnerabilities and enhance their capacities. Ref. [6] internationally, the trend continues to build capacity in government disaster management capabilities and functions in developing nations and to promote community-based hazard mitigation planning and programs.

The use of new technologies like smart phones and web application play a big role in improving emergency system. Mobile devices have become increasingly important in the developing world, facilitating communication between locals, government officials and first responders [7]. Many applications provide important information in areas of health, agriculture, disaster relief, and crime.

The mass communications media not only quickly notifies the world of disastrous events, but many times their versions are greatly dramatized, if not distorted. In addition, news reports usually do not give specific information about the exact location of a disaster, or details to indicate who has or has not been involved. But with the use of geolocation, the location is plotted on the map and user's information will be sent to the command center [8].

Many of the emergency systems exists are landline-based. With the fast development in technology, especially the emergence of smartphones where almost many people hand carries these devices; we propose a system that would give people an alternative and added option or medium in calling for rescue. Providing the people a mobile application to be installed on their smart phones to send emergency requests and a web application to be deployed on command centers to receive and locate the mobile app users, this might be useful for easy and fast dispatching of emergency units.

\section{PROPOSED SYSTEM}

\section{A. Technologies Involved}

The core concept of the researchers focuses on a mobile 
and a web application. Our major goal is to provide information such as name, age, type of emergency response needed and location of a person using the mobile app and to be retrieved by a system on the web and plotting the equivalent latitude and longitude on a Google map in order to pinpoint the exact location of the person who uses the mobile app. To achieve it, the researchers also studied an extensive array of technologies focusing on computer engineering field.

For the mobile application, the researchers plan to use Phonegap instead of a native android language in order to maximize the user interface and to make it flexible and easy for other mobile platforms to adopt. Phonegap is a free and open source framework that allows you to create mobile apps using standardized web APIs for different mobile platforms. Basically it uses HTML, CSS and JavaScript, and wraps it with phonegap then deploy to different mobile operating systems like Android, iOS, Windows, Windows 8, Tizen, Blackberry, Blackberry 10.

QuoJS will be used as the main JavaScript Library for the mobile application. It is a micro, modular, Object-Oriented and concise JavaScript Library that simplifies HTML document traversing, event handling, and Ajax interactions for rapid mobile web development. It allows writing powerful, flexible and cross-browser code with its elegant, well documented and micro coherent API.

For getting the user's location, it requires to have a stable internet connection and an enabled GPS for more accurate reading of latitude and longitude points. Google Maps JavaScript API will be used to read user's geolocation. Reverse geocoding will also takes place in order to convert the geographic coordinates and display a human readable address to the user.

Since the researchers will focus more on running the mobile app on an android platform, Fries framework will be used. It is a mobile UI development framework that creates a native android-like feel using HTML, CSS and JavaScript.

For the web application, the researchers intend to use SailsJs. It is a MVC Framework for Node.js. Node.js is a platform built on Chrome's JavaScript runtime for easily building fast, scalable network applications. Node.js uses an event-driven, non-blocking I/O model that makes it lightweight and efficient, perfect for data-intensive real-time applications that run across distributed devices. We will also use Socket.io. Socket.IO aims to make real-time apps possible in every browser and mobile device, blurring the differences between the different transport mechanisms. It's care-free real-time $100 \%$ in JavaScript.

Tuktuk is the main UI framework for the web app. It is simple and a Responsive Web Design framework for creating websites and web applications. It contains HTML and CSS-based design templates for typography, forms, buttons, navigation and other interface components.

All the data will be stored on a MongoDB database. It is a NoSql and a document database that provides high performance, high availability, and easy scalability.

\section{B. System Architecture}

In the initialization of the mobile application, it detects the current position of the user through geolocation. The user can navigate in three tabs namely home, info and hotlines. The home tab contains the current location of the user. It is displayed on the map. Geographical points are converted into human-readable address. Three emergency buttons are present: ambulance, police and fire truck. The info tab contains details like name, age and mobile number of the user. He/She needs to input once and data will be save but he/she can edit if necessary. The hotlines tab contains other emergency hotlines. Since the application is internet dependent because of the geolocation, the emergency numbers are pre dialled enabling the user to call for emergency even without an internet. The system architecture for the mobile application is designed as described in Fig. 1.

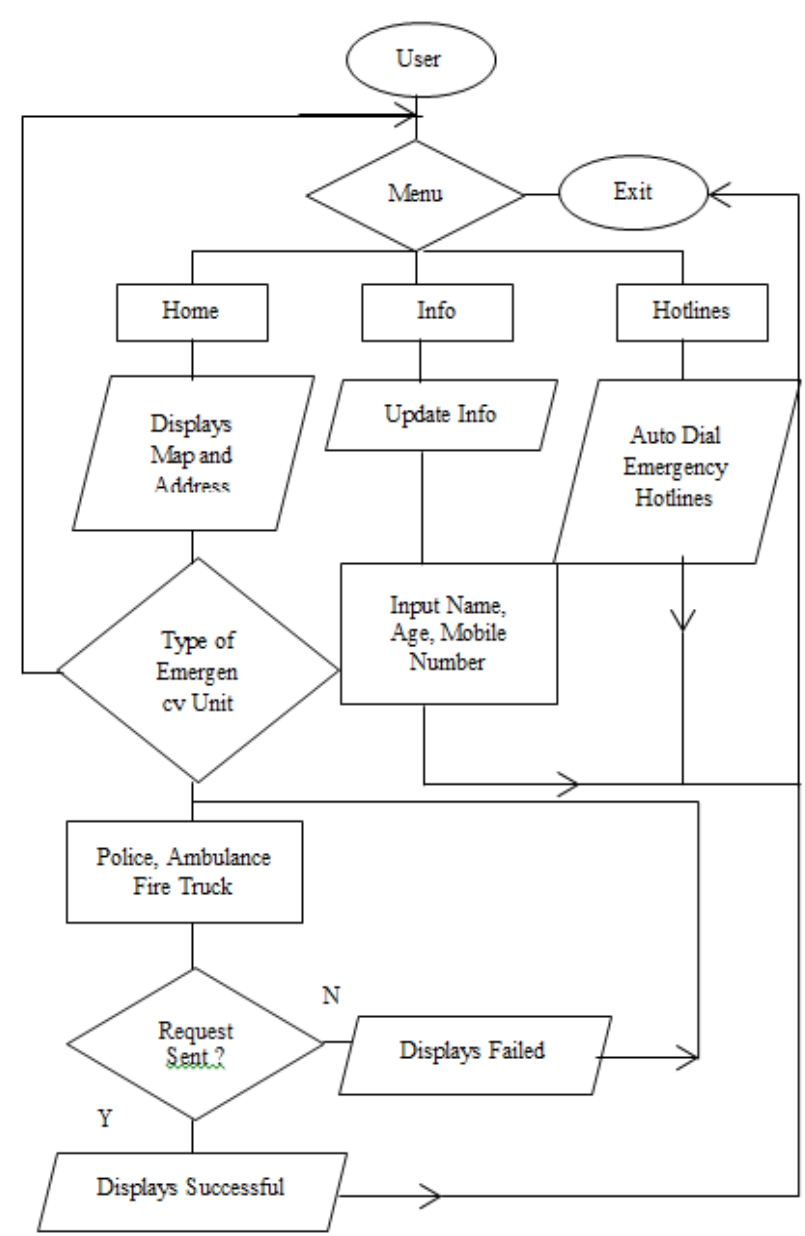

Fig. 1. System Architecture of the mobile application.

For the web application, the dashboard contains a map and a side bar where emergency reports are appended real time. The user can respond, decline and view the report on the map. The system architecture for the mobile application is designed as described in Fig. 2.

\section{Graphical User Interface}

The researchers provide graphical user interfaces for both mobile and web application where the users can interact with the emergency response system. Fig. 3 shows the prototype of the home tab of the mobile application where the users can select emergency request for ambulance, police and fire truck.

Fig. 4 shows the prototype of the info tab of the mobile application where the users can update their personal information that will be send to the command center. 


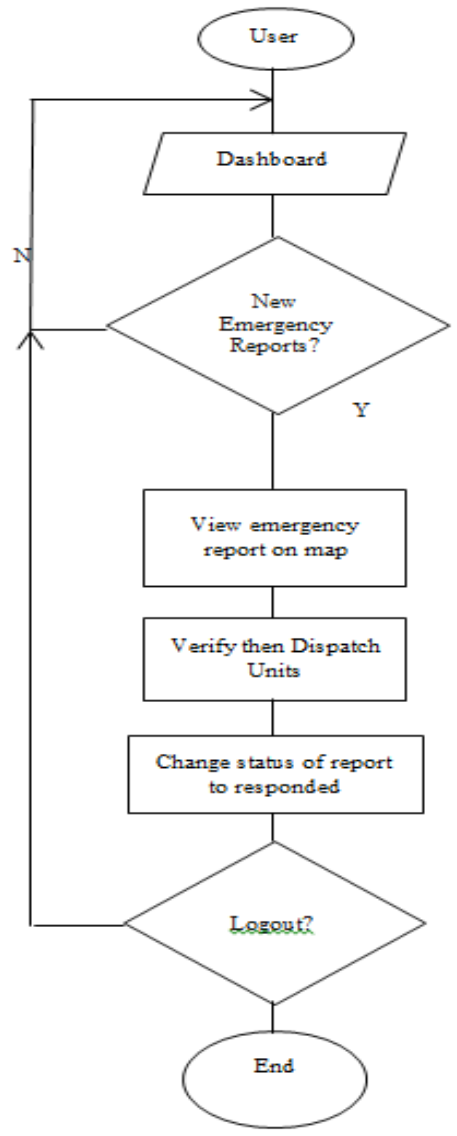

Fig. 2. System architecture of the web application.

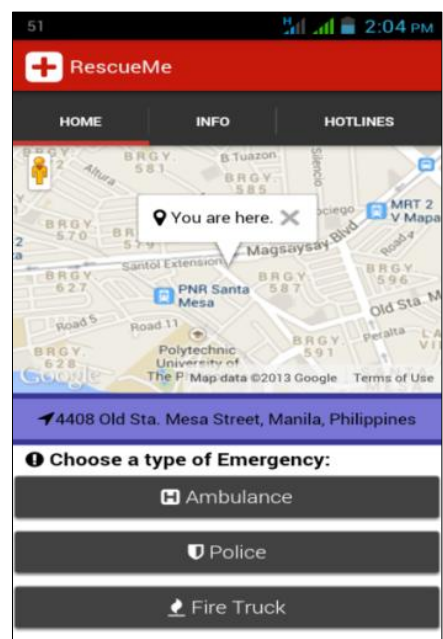

Fig. 3. Home tab.

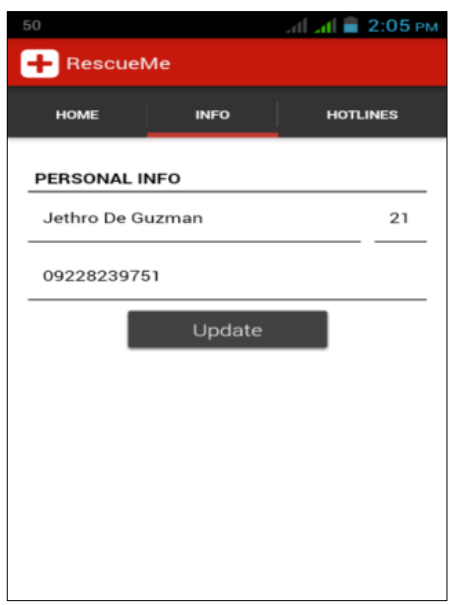

Fig. 4. Info tab.

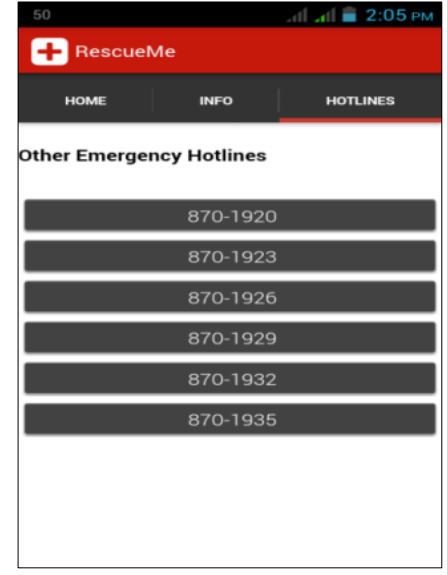

Fig. 5. Hotlines tab.

Fig. 5 shows the prototype of the hotlines tab of the mobile application where it contains pre dialled hotline numbers that can be used when the application is not connected in the internet.

Fig. 6 shows the prototype of the dashboard of the web application where it retrieves the sent emergency request and plot on the map the location from the mobile app user.

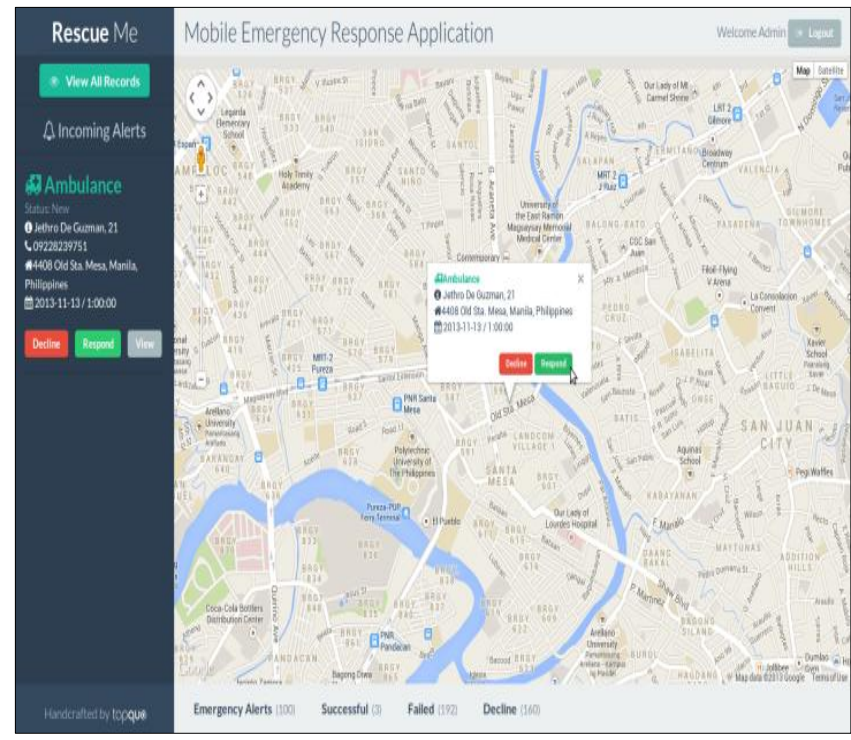

Fig. 6. Web application dashboard.

\section{SCOPE AND LIMITATION}

The study is mainly focused on the development of a mobile emergency application for the community and a web application for Command Centers.

The mobile application can only run on devices particularly smart phones that run on an Android Operating System version 2.3 Gingerbread or Higher for the meantime. But the app can be ported to other platform since it will be created using Phonegap. While the web application, it can be accessed through the internet.

The mobile app can be downloaded by the community from a webpage where the web application is also hosted. The application basically would detect the user's current location. The user needs to fill up some personal information for verification, such as name, age and mobile number. The application is only limited into three emergency units such as ambulance, police and fire truck. 
Once the user click to request a certain emergency unit, the application automatically send the user's geographical points using the phone's GPS together with the personal information. The Command Center's web application will retrieve the sent distress request from the mobile app and plot it in Google Maps real-time.

Since the mobile app needs to track the location of the user, it requires a stable internet connection. Without it, the application won't be able to send an emergency request. Because the application would rely to the IP address provided by the internet connection to locate the current position of the user in the map. The accuracy of the position's detection of the user also depend on the place and how stable the user's internet connectivity. The error of the GPS position is mainly determined by the interaction of the time varying constellation of the satellites and the built-up in the close vicinity [9]. The average position error ranges from 2 meters on an open square to 15 meters even in wide streets with four story houses on both sides. The built-up shades the satellites especially suitable for a positioning. The constellation of the satellites is periodic and the built-up constant, therefore a rudimentary database was used to reduce the positioning error by $\sim 10 \%$. We will also provide auto dialled links for other emergency hotlines in case the user won't have a chance to connect to the internet.

The mobile app cannot support location tracking for users on a moving vehicle. The app detects the last position of the user on the map, and that is the location to be sent to the command center.

\section{CONCLUSIONS}

In this paper, we proposed the use of mobile and web technologies to add another option and medium for emergency response. The proposed method used the current trends in mobile and web technologies for fast and efficient dispatching of emergency units

Our goal is not to create a new protocol in emergency response, we have just maximize the use of smart phones to act as medium and to help people save their lives in case of disaster. Command centers will also benefit in a way that the location of the user are easily detected and plotted on a map.

Our porposed system supposed to lessen the response time it takes to respond to emergency events. It also provides reliable information that might help in identifying accidents.

\section{REFERENCES}

[1] Emergency Response. [Online]. Available: http://www.niehs.nih.gov/health/topics/population/response/

[2] About the national 911 office. [Online]. Available: http://www.911.gov/about.html

[3] People's action team responding on-line (PATROL 117). [Online]. Available:

http://www.lgrrc6.org.ph/index.php?option=com_content $\&$ view=artic le\&id=127:patrol117\&catid=35:programs \&Itemid $=2$

[4] J. Therese, B. Fajardo, and C. M. Oppus, "A mobile disaster management system," International Journal of Communications, vol. 3, issue 3, 2009.

[5] M. Victoria and G. Pineda, "Exploring the potentials of a communitybased disaster risk management system, the Philippine experience," International Journal of Innovation, Management and Technology, vol.3, no.6, December 2012.

[6] Emergency and risk management case study. [Online]. Available: http://training.fema.gov/EMIWeb/edu/emoutline.asp

[7] D. M. West and E. Valentini, "How mobile devices are transforming disaster relief and public safety," Issues in Technology Innovation, p. 5, July 2013.

[8] E. A. der Heide, "Disaster response: principles of preparation and coordination," CV Mosby St. Louis.

[9] M. Modsching, R. Kramer, and K. T. Hagen, "Field trial on GPS accuracy in a medium size city: The influence of builtup," University of Applied Sciences Zittau/GÄrlitz, Germany

[10] G. O. Young, "Synthetic structure of industrial plastics," in Plastics, 2nd ed. vol. 3, J. Peters, Ed., New York: McGraw-Hill, 1964, pp. 1564.

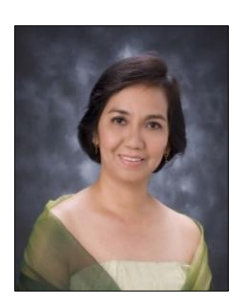

Engr. Remedios G. Ado obtained her master degree of engineering computer. She is designated currently, the assistant director of PUP - Information and Commnication Technology Office (ICTO). She was the former chairperson of master of science in information technology of the PUP Graduate School and Open University and chairperson of Computer Engineering Department in addition to her duties as the associate professor. Her research undertaken now is: "Fabrication and Application of Interface Circuits to the microprocessor" in Japan.

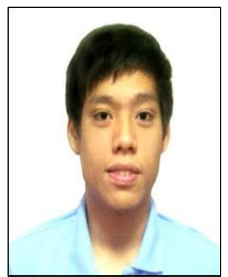

Jethro B. De Guzman is a $5^{\text {th }}$ year BS student majoring in computer engineering of Polytechnic University of the Philippines. Knowledgeable in web programming and frameworks (HTML, CSS, JS, PHP, laravel, sailsjs, MySQL, MongoDB, JQuery). He took his intership in Codelean and 8Layer Technologies both as junior web developer.

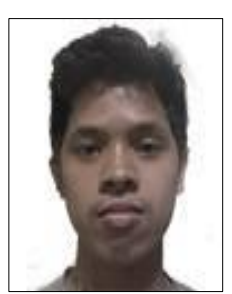

Ritz Carlo C. D Guzman is a $5^{\text {th }}$ year BS stuent majoring in computer engineering of Polytechnic University of the Philippines. $\mathrm{He}$ is knowledgeable in programmming languages (Java, HTML, CSS, PHP, JS). 